\title{
Loudness and the binaural masking level difference
}

\author{
D. R. SODERQUIST and R. D. SHILLING \\ University of North Carolina at Greensboro, Greensboro, North Carolina
}

\begin{abstract}
The large threshold difference found between a low-frequency antiphasic signal, $\mathrm{S} \pi$, and inphase signal, SO, in the presence of a homophasic noise masker (NO) is referred to as the binaural masking level difference (BMLD). The magnitude of the BMLD diminishes when the antiphasic signal is increased to suprathreshold levels. The decrease in effectiveness of a $180^{\circ}$ phase-shifted signal, at suprathreshold levels, produces a concomitant decrease in perceived loudness of the signal. This decrease in effectiveness at the higher signal levels was examined in a loudnessmatching paradigm. A change in neural processing was hypothesized to account for the variation in loudness, detectability, discrimination, and identification when the signal level approached $20 \mathrm{~dB}$ SL.
\end{abstract}

An interaural in-phase low-frequency sinusoid (designated SO), when presented simultaneously with a moderately intense in-phase interaural noise (NO), yields a significantly higher masked threshold than does an out-of-phase sinusoid $(\mathrm{S} \pi)$ masked by the identical noise. The difference in decibels between the masked threshold in the former condition (NOSO) relative to the threshold in the antiphasic condition (NOS $\pi$ ) is the binaural masking level difference (BMLD). This release from masking has an extensive literature and has been well documented (e.g., Green \& Henning, 1969; Small et al., 1972; Soderquist \& Lindsey, 1970).

An interesting aspect of the BMLD is that it is a nearthreshold phenomenon. When one considers the loudness of the two signals at suprathreshold levels, the effect of interaural time/phase has diminished. For example, at masked threshold (0-dB sensation level, SL), the two signals are equally loud. If, however, each signal, SO and $\mathrm{S} \pi$, is increased $10 \mathrm{~dB}$ above its respective masked threshold, a clear discrepancy in loudness occurs. The $S \pi$ signal is perceptually less loud than SO, even though each signal is $10 \mathrm{~dB}$ above its masked threshold. To be equally loud, the $S \pi$ signal must be increased by more than $10 \mathrm{~dB}$. This is most likely due to the decreasing effectiveness of the NOS $\pi$ interaction at suprathreshold levels.

The only systematic investigation concerning this particular point of diminishing effectiveness appears to be that of Townsend and Goldstein (1972). These investigators used the method of adjustment to show that, for various signal levels at different noise spectrum levels, the loudness differences between SO and $S \pi$ tend to disap-

This research was supported, in part, by a grant to D. R. Soderquist from the National Institute of Neurological and Communicative Disorders and Stroke (1 R15 NS23187-01A1). Reprint requests may be addressed to D. R. Soderquist, Department of Psychology, University of North Carolina at Greensboro, NC 27412, or via electronic mail: DRS@UNCG.bitnet. pear when the SO signal has increased to $20 \mathrm{~dB}$ SL. The present study expands on these data by examining additional signal frequencies and using a two-alternative forced-choice (2AFC) adaptive procedure to procure the BMLD.

Subjects

\section{METHOD}

There were two well-trained subjects, the senior author and a student. Both subjects had normal hearing in the frequency ranges used.

\section{Apparatus and Stimulus}

The subject was seated in a double-walled sound-attenuating chamber and listened to stimuli through matched earphones (TDH-49) mounted in supraural cushions (MX41/AR). A response panel with lights and buttons was used to indicate when to listen and when to respond. Feedback was given in the BMLD task but not for loudness-matching conditions. The signals were digitally generated using ILS-PC software on an IBM-AT microcomputer. The 300-msec signals had linear rise/fall ramps of $10 \mathrm{msec}$ each and were converted by a Data Translations DT2801A D/A converter at a $25-\mathrm{kHz}$ sampling frequency. Signal frequencies were $200,300,400,500,750$, and $1000 \mathrm{~Hz}$. Two levels of analog noise (15- and 30-dB spectrum level) were produced by a HewlettPackard 8057-A precision noise generator. The noise was bandpassed through a 48-dB/octave Coulbourn Instruments S75-36 variable filter $(100-1100 \mathrm{~Hz}, 3 \mathrm{~dB}$ down), and shaped (10 msec rise/decay) with a Coulbourn S84-04 rise/fall module. Stimuli were mixed with a Coulbourn Instruments mixer/amplifier (S82-24) and attenuated with programmable Coulbourn Instruments attenuators (S85-08). A Crown D-75 amplifier was also used in the noise circuitry. Stimuli were monitored with a Ballantine 320A true RMS meter, Tektronix storage scope/spectrum analyzer (5A15N and 5L4N, respectively), and Hewlett-Packard 5221B electronic counters and manual attenuators (350-D). Interaural phase shift of the signal was created with a Grason-Stadler phase shifter (Model E3520B). The experiments were under the control of a locally written Turbo Pascal program and the IBM-AT microcomputer.

\section{Procedure}

The experiment was conducted in two stages. Stage 1 was the determination of the BMLD using a 2AFC 2-down and 1-up adaptive psychophysical method. This procedure yields a $71 \%$ correct detection at the masked threshold (Levitt, 1971). Thresholds for the six signal frequencies were obtained for the NOSO and NOS $\pi$ conditions at each spectrum level of the noise. Each trial consisted of two presentations of a 500 -msec noise with a feedback light following the subject's response. 
The 300-msec signal, when it occurred, was temporally located in the center of the noise burst. The signal was presented, at random, in one of the two listening intervals. The suprathreshold starting level of the signal was chosen randomly for each run. The signal decreased or increased in 3-dB steps until the third turnaround and in a 1-dB step thereafter. Final masked threshold was based on the mean of three runs, with the threshold estimate for each run in turn determined by averaging the signal level associated with the last six turnarounds. A practice run preceded each block of three runs.

Stage 2 was a loudness-matching procedure using a variation of the classical method of adjustment. Each trial consisted of a 1,500-msec noise burst and two signals. The first signal, the standard, was always a $300-\mathrm{msec}, 1000-\mathrm{Hz}$ in-phase sinusoid. It was pulsed on $300 \mathrm{msec}$ after the noise onset. The second signal, the comparison signal, followed the standard by $300 \mathrm{msec}$. The noise terminated $300 \mathrm{msec}$ after the comparison signal. The comparison signal was, for each run, either interaurally in-phase $\left(0^{\circ}\right)$ or $180^{\circ}$ out-of-phase, and was one of the six frequencies used in Stage 1. The subject's task was, on each trial, to adjust the level of the comparison signal in 1-dB steps by pressing one of two buttons. Once the level of the comparison approached the point where it was perceived as equal in loudness to the standard, the subject was instructed to bracket the "equally loud point." As in the BMLD study, the mean of each run was based on the last six turnarounds generated by the bracketing procedure. The final loudness level was based on the estimates from three runs. The standard had a sensation level of 10 , 20 , or $30 \mathrm{~dB}$ relative to the subject's $1000 \mathrm{~Hz}$ in-phase masked threshold obtained in Stage 1.

\section{RESULTS}

Figures 1 and 2 show the results for each subject. In both figures, filled symbols represent the signal level, in decibels SPL, for the NOS $\pi$ condition. The open symbols are the signal levels in SPL for the NOSO conditions. The disparity between symbols of the same shape reflects the difference in signal level (dB SPL) when SO and $S \pi$ were equally loud. The differences at $0 \mathrm{~dB}$ SL reflect both equal loudness and the BMLD obtained in Stage 1.

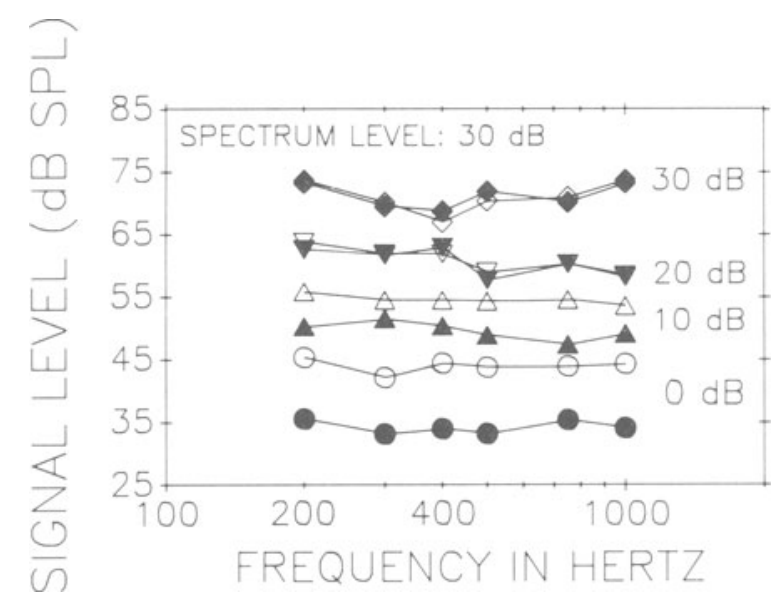

Figure 1. Equal-loudness contours as a function of signal frequency in the presence of a 30-dB-spectrum-level noise. Filled symbols represent the $S \pi$ signals; open symbols are SO signals. Signals represented by the same-shape symbols are equally loud and have the same loudness as the standard. Inverted triangles, standard $20 \mathrm{~dB}$ SL; upright triangles, standard $10 \mathrm{~dB}$ SL; circles, BMLD data. Subject D.S.

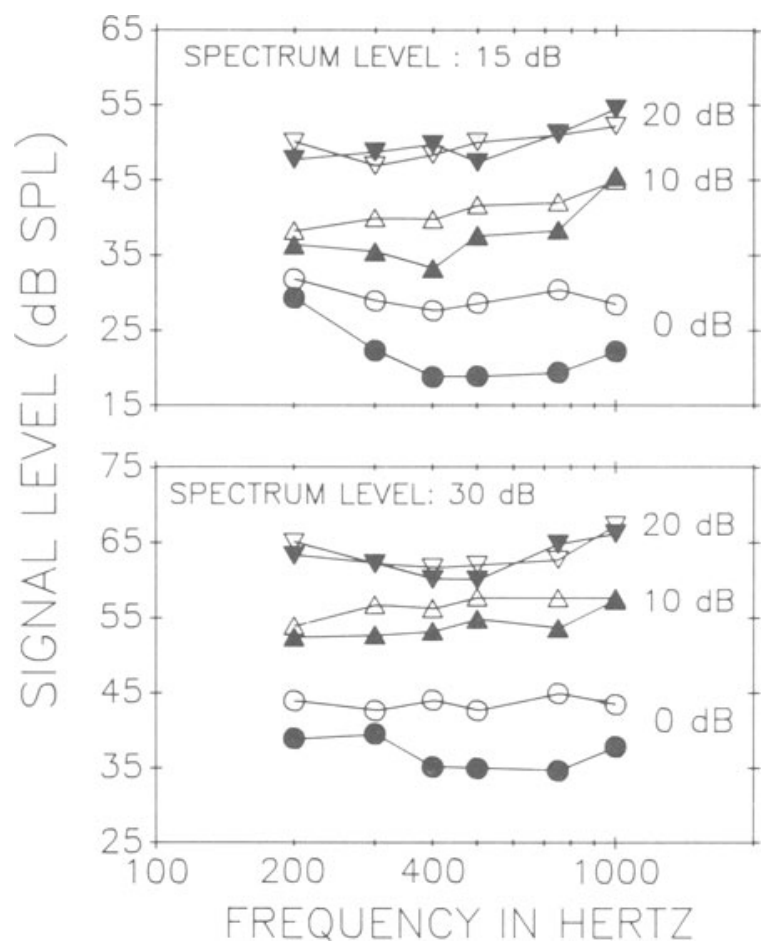

Figure 2. Equal-loudness contours as a function of signal frequency in the presence of either a 15- or 30-dB spectrum-level noise. Filled symbols represent the $S \pi$ signals; open symbols are SO signals. Signals represented by the same-shape symbols are equally loud and have the same loudness as the standard. Inverted triangles, standard $20 \mathrm{~dB}$ SL; upright triangles, standard $10 \mathrm{~dB}$ SL; circles, BMLD data. Subject K.V.

The data shown in the figures clearly reveal that the $\mathrm{S} \pi$ signal loses its effectiveness as its level increases to suprathreshold values. For example, at $0 \mathrm{~dB}$ SL, the SO and $S \pi$ signals are equally loud (because both have been matched in loudness to the standard) even though they differ considerably in intensity (dB SPL). At suprathreshold levels, however, when the two signals have the same loudness, the effect of the interaural phase shift of the $S \pi$ signal has diminished. In the latter condition the $S \pi$ signal must be increased in level to be perceived as equal in loudness to the standard and, therefore, also equal in loudness to the SO signal. For example, when the comparison signal, either SO or $S \pi$, is matched in loudness to the standard, each comparison signal has the same intensity level when the standard is $20 \mathrm{~dB}$ SL. In sum, the interaural phase shift of the signal, which yields the decrease in signal threshold for BMLD conditions, systematically loses its effectiveness as the signal levels increase above masked threshold.

\section{DISCUSSION}

As noted in the introduction and as demonstrated in the results, the antiphasic condition leads to a distinct improvement in detectability. In addition, there are the extant data which show that the NOS $\pi$ condition also reduces the frequency difference limen and improves identifica- 
tion (e.g., Gebhardt \& Goldstein, 1972; Licklider, 1948). A plausible hypothesis to explain the current data, and the discrimination and identification results, begins by considering the neural output from each cochlea as a function of the NOSO and NOS $\pi$ conditions. In the following discussion, it is assumed, quite logically, that the rate of neural activity, at masked theshold, is different for the homophasic and antiphasic conditions. The antiphasic condition, because of the decreased signal level, produces a lower rate of neural activity than does the homophasic condition.

Consider now the NOS $\pi$ condition at masked threshold. It is hypothesized that the neural firing pattern from each ear produces an interaural temporal "disparity" at a central location. The temporal disparity is the difference in the neural firing patterns received from each cochlea. The disparity is processed by higher neural centers and promotes the lower behavioral thresholds and improved discrimination and identification. It is further suggested that, at masked threshold, the critical band at each ear integrates the noise and the antiphasic signal. The output of the integration is sent to the central auditory processor (i.e., medial superior olive) as a neural pattern of activity containing temporal information. The temporal neural pattern coming from each critical band is constantly changing as a function of the interaural variability inherent in the noise and signal. Therefore, the disparity is also changing continuously in time. At low $\mathrm{S} / \mathrm{N}$ ratios, then, the disparity is predominant at the central processor and the behavioral thresholds are improved. However, when the antiphasic signal is suprathreshold ( $\mathrm{S} / \mathrm{N}$ ratio increases), the signal effectively "swamps" the noise components within the critical band and the integration becomes weighted toward the signal. The overall rate of neural firing becomes the principal factor in detection, discrimination, identification, and loudness. In this latter case, temporal disparity reverts to a minor role. In sum, at suprathreshold, when the signal dominates, the noise components are effectively removed as part of the temporal pattern and the disparity is reduced. Even though the suprathreshold $\mathbf{S} \pi$ signal produces temporal pattern differences (assuming the signal is phase-locked), the disparity is less variable and the overall rate of neural activity becomes the salient feature in the neural processing. The result is that at suprathreshold levels the auditory system uses the overall rate of neural activity to determine behavioral thresholds and to make loudness matches. In the NOSO condition, on the other hand, behavioral thresholds and loudness matches can be based only on the overall rate of activity; there is no temporal disparity between the two cochlear inputs to the central processor. Finally, when the rate of firing in the NOS $\pi$ condition is equivalent to the rate of firing in the NOSO condition, the behavioral thresholds and loudness are the same. The shift from disparity to rate is complete when the signal, in the NOS $\pi$ condition, approaches $20 \mathrm{~dB}$ SL.

\section{REFERENCES}

Gebhardt, C. J., \& Goldstein, D. P. (1972). Frequency discrimination and the M.L.D. Journal of the Acoustical Society of America, 51, 1228-1232.

Green, D. M., \&enning, G. B. (1969). Audition. Annual Review of Psychology, 20, 105-128.

LEVITT, H. (1971). Transformed up-down methods in psychoacoustics. Journal of the Acoustical Society of America, 49, 467-477.

LICKLIDER, J. C. R. (1948). The influence of interaural phase upon the masking of speech by white noise. Journal of the Acoustical Society of America, 20, 150-159.

Small, A. M., Boggess, J., Klich, R., Kuehn, D., Thelin, J., * WILEY, T. (1972). MLD's in forward and backward masking. Journal of the Acoustical Society of America, 51, 1365-1367.

SoDERQUist, D. R., \& LiNDSEY, J. W. (1970). Masking-level differences as a function of noise spectrum level, frequency, and signal duration. Journal of Auditory Research, 10, 276-282.

TOWNSEND, T. H., \& GolDSTEIN, D. P. (1972). Suprathreshold binaural unmasking. Journal of the Acoustical Society of America, 51, 621-624.

(Manuscript received April 18, 1990.) 